\title{
INVESTIGACIÓN
}

Recibido: 16/04/2021 --- Aceptado: 31/08/2021 --- Publicado: 24/09/2021

\section{EDUCANDO AL HOMO DIGITALIS: EL PAPEL DE LA EDUCACIÓN Y DEL DIGCOMEDU PARA PALIAR LOS EFECTOS DE LOS ALGORITMOS, LAS FAKE NEWS, LA POLARIZACIÓN Y FALTA DE PENSAMIENTO CRÍTICO}

Educating the homo digitalis: the role of education and digcomedu to palyate the effects of algorithms, fake news, polarization and lack of critical thinking

Eva Teba Fernández. Universidad Camilo José Cela. Madrid, España. eteba@ucjc.edu

Cómo citar el artículo:

Teba Fernández, E. (2021). Educando al homo digitalis: el papel de la educación y del digcomedu para paliar los efectos de los algoritmos, las fake news, la polarización y falta de pensamiento crítico. Vivat Academia. Revista de Comunicación, 154, 71-92. http:/ / doi.org/10.15178/va.2021.154.e1378

http://www.vivatacademia.net/index.php/vivat/article/view/1378

\section{RESUMEN}

Las aulas de nuestras organizaciones educativas (escuelas, centros de secundaria y universidades) están habitadas por integrantes de las llamadas "generación de los millenials”, “generación Z" y "generación Alfa”, niños, adolescentes y jóvenes nacidos en una sociedad aumentada donde la tecnología y las redes sociales están omnipresentes y donde los algoritmos toman cada vez más decisiones o sesgan sus percepciones. El impacto que la hiperconexión y la corporeización de las tecnologías tiene en sus vidas alcanza diversos grados, dependiendo de la generación a la que pertenezcan, pero puede causar serias disfunciones psicológicas, relacionales y dilemas éticos. Además, la generalización de las tecnologías en el mundo educativo nos está llevando, por un lado, a nuevas formas de poder y control de la sociedad, pero también a nuevas brechas de exclusión. ¿Qué papel ha de cumplir la educación en este escenario? ¿La formación inicial y permanente de los docentes está atendiendo estas cuestiones? El artículo revisa estas cuestiones y propone un escenario formativo que parte del DigComEdu, el Marco Europeo para la Competencia Digital de los Educadores.

PALABRAS CLAVE: Competencia digital - Alfabetización digital - Formación del profesorado - Tecnología educativa - Brecha ética digital - FoMO - Identidad digital - Sesgo de confirmación - Algoritmo.

\section{ABSTRACT}


Educando al homo digitalis: el papel de la educación y del digcomedu para paliar los efectos de los algoritmos, las fake news, la polarización y falta de pensamiento crítico

The classrooms of our educational organizations (primary schools, secondary schools and universities) are inhabited by members of the so-called "Millennials", "generation Z" and "generation Alpha", generations made up of children, adolescents and young people born in an augmented society where technology and social media are ubiquitous and where algorithms increasingly make decisions or skew their perceptions. The impact that hyperconnection and the embodiment of technologies has on their lives reaches varying degrees, depending on the generation to which they belong, but it can cause serious psychological and relational dysfunctions and ethical dilemmas. Furthermore, the generalization of technologies in the educational world is leading us, on the one hand, to new forms of power and control of society, but also to new exclusion gaps. What role should education play in this scenario? Is the initial and ongoing training of teachers addressing these issues? The article reviews these issues and proposes a training scenario based on DigComEdu, the European Framework for Educators' Digital Competence.

KEYWORDS: Digital competence - Digital literacy - Teacher training - Educational technology - Digital ethics gap - FoMO - Digital identity - Confirmation bias Algorithm.

\section{EDUCANDO HOMO DIGITALIS: O PAPEL DA EDUCAÇÃO E DO DIGCOMEDU PARA PALIAR OS EFEITOS DE ALGORITMOS, FALSAS NOTÍCIAS, POLARIZAÇÃO E FALTA DE PENSAMENTO CRÍTICO.}

\section{RESUMO}

As salas de aula das nossas organizações educacionais (escolas, escolas secundárias e universidades) são habitadas por membros da chamada "geração dos millennials", "geração Z" e "geração Alfa", crianças, adolescentes e jovens nascidos em uma sociedade aumentada onde a tecnologia e as mídias sociais são onipresentes e os algoritmos cada vez mais tomam decisões ou distorcem suas percepções. O impacto que a hiperconexão e a incorporação de tecnologias têm nas suas vidas atinge graus variáveis, dependendo da geração a que pertencem, mas pode causar graves disfunções psicológicas e relacionais e dilemas éticos. Além disso, a generalização das tecnologias no mundo educacional está nos levando, por um lado, a novas formas de poder e controle da sociedade, mas também a novas lacunas de exclusão. Qual deve ser o papel da educação nesse cenário? O treinamento inicial e contínuo de professores aborda essas questões? O artigo analisa essas questões e propõe um cenário de treinamento com base no DigComEdu, o European Framework for Educators 'Digital Competence.

PALAVRAS CHAVE: Competência digital - Literacia digital - Formação de professores - Tecnologia educacional - Lacuna de ética digital - FoMO - Identidade digital - Viés de confirmação - Algoritmo. 
Educando al homo digitalis: el papel de la educación y del digcomedu para paliar los efectos de los algoritmos, las fake news, la polarización y falta de pensamiento crítico

\section{INTRODUCCIÓN Y ESTADO DE LA CUESTIÓN}

Actualmente estamos siendo testigos de profundas transformaciones sociales, geopolíticas, económicas y culturales, que se anunciaban desde finales del siglo XX y que se han acelerado en las dos primeras décadas del s. XXI. No podemos obviar el innegable avance de la Humanidad desde el último tercio del pasado siglo, lo que ha producido una falsa sensación de omnipotencia sobre los destinos del Planeta, como nos recuerda el historiador Yuval Harari en sus obras Sapiens (2014) y Homo Deus (2016). Una ficción que se ha destruido en pedazos debido a la pandemia de COVID19 en la que aún estamos inmersos en el momento de escribir este artículo.

Pero incluso antes de la zoonosis que ha puesto al coronavirus en nuestras vidas, Warren Bennis y Burt Nanus (1985) ya habían hablado a finales del siglo XX de la necesidad de que las organizaciones y las personas se prepararan para adaptarse de forma ágil y flexible a los entornos VUCA (Volatility, uncertainty, complexity and ambiguity), incertidumbre y volatilidad que tienen una cara negativa, bautizada por Zygmunt Bauman como "modernidad líquida" (2007): un mundo en el que la identidad de los individuos se construye como consumidores al acecho de una felicidad inmediata e irreflexiva y de la recompensa inmediata. Bombardeados por la infodemia continua y sesgada, las personas han perdido contacto con todas las referencias ideológicas, sociales y de comportamiento moral y ético que habían conducido sus actuaciones en épocas anteriores.

En estos últimos tres años, la educación mundial ha generado nuevos marcos, de enfoque holístico y más humanista, que respondan a las necesidades complejas de un Planeta cada vez más global e interconectado. El objetivo común que todos persiguen es minimizar los aspectos negativos de una innovación y cambio tecnológico que tienen un historial de contribución desigual en el florecimiento humano, como hemos podido comprobar en el muy desigual efecto que la pandemia ha tenido en los países más y menos desarrollados del mundo durante 2020. La brújula para el aprendizaje del 2030 de la OCDE (2018, 2020), La iniciativa Futuros de la Educación 2050 de la UNESCO (2019a, 2019b) o el Pacto Educativo Global promovido por el Papa Francisco desde el Vaticano (2020) son solo tres de los ejemplos más significativos de estos marcos, que buscan ese papel transformador de la educación para el Bien Común y el logro del Bienestar humano. Estamos inmersos en una cuarta revolución industrial que ha cambiado las formas de trabajar, de producir bienes, de relacionarnos, e incluso las competencias y destrezas que más se van a valorar en los próximos años a nivel laboral, entre las que el pensamiento crítico, del que hablaremos en este artículo, tiene una importancia primordial (World Economic Forum, 2020). Por ello insistimos que el mundo educativo debe continuar con la reflexión para entender si está afrontando adecuadamente sus planteamientos didácticos y marcos curriculares, incluido el uso de la tecnología, para garantizar que está preparando a los educandos de hoy para el mundo del presente y del futuro.

Vivat Academia. Revista de Comunicación. 2021, 154, 71-92 
Educando al homo digitalis: el papel de la educación y del digcomedu para paliar los efectos de los algoritmos, las fake news, la polarización y falta de pensamiento crítico

Desde la llegada de Internet, el uso de las tecnologías de la información y la comunicación han ido transformando, sin que nos diéramos apenas cuenta, nuestra realidad cotidiana y educativa. El gran salto se ha producido, sobre todo, desde que aparecieron los dispositivos móviles, sean tabletas o teléfonos inteligentes, que nos convierten en una especie de "cyborgs posmodernos" (Sánchez y Andrada, 2013), siendo un apéndice más de nuestro cuerpo en los que delegamos cuestiones como nuestra capacidad de orientación -aplicaciones como Google Maps- y transforman incluso nuestra expresión lingüística - de nuestro smartphone decimos "me he quedado sin batería" en lugar de "se ha quedado sin batería"-. Nadie discute las ventajas que puede tener la incorporación de la tecnología en el mundo educativo, máxime en medio de una emergencia sanitaria como la actual pandemia generada por la COVID19. Sin esta incorporación cotidiana de la tecnología en nuestras vidas, hubiera sido inviable lo que se ha etiquetado como "enseñanza remota de emergencia" que, a pesar de sus carencias, ha permitido que los procesos de enseñanza-aprendizaje no quedaran súbitamente interrumpidos durante los periodos de confinamiento domiciliario a los que nos hemos visto obligados en los distintos países del mundo. Sin embargo, "enseñanza remota de emergencia" no es sinónimo de una adecuada integración de las tecnologías educativas ni de un buen diseño instruccional, por no hablar de otras dimensiones, como la salud digital y la alfabetización mediática, en las que se centra este artículo. Uno de los factores clave de una adecuada integración de la tecnología educativa es la calidad de la formación que recibe el profesorado, y en lo que respecta a la alfabetización digital crítica y la lucha contra la desinformación en la red, aún nos queda camino por hacer. El impacto que la hiperconexión, la corporeización y la excesiva dependencia de las tecnologías tiene en el alumnado que habita nuestras instituciones educativas alcanza diversos grados, dependiendo de su edad y grado de madurez, pero hay investigación abundante y reciente (Echeburúa y de Corral, 2010; Franchina et al, 2018; Overton, 2019; Terán Prieto, 2019; Torres Serrano, 2020) que demuestra puede causar serias disfunciones psicológicas, relacionales y dilemas éticos. La educación es básica para ayudar al alumnado a evitar la manipulación intelectual y emocional, a mantener una autoestima fuerte, a pesar de las influencias externas, y a construir un autoconcepto más sólido que les ayude a evitar sentimientos de exclusión, de inferioridad y de trastornos de ansiedad y depresión.

\section{METODOLOGÍA}

El presente artículo realiza una revisión de literatura reciente, que informa la posterior discusión argumentada. Algunos de los constructos que usamos para dicha discusión (Sobreexposición y sobreidentificación) provienen de las categorías de conductas problemáticas de la publicación Factores de socialización digital juvenil de la Fundación de Ayuda para la Drogadicción (Gordo et al., 2019).

El método de revisión bibliográfica se concreta en búsqueda en bases de datos digitales utilizadas en educación (ERIC y Dialnet), así como en revistas especializadas en formación del profesorado de España y Latinoamérica.

Vivat Academia. Revista de Comunicación. 2021, 154, 71-92 
Educando al homo digitalis: el papel de la educación y del digcomedu para paliar los efectos de los algoritmos, las fake news, la polarización y falta de pensamiento crítico

Si bien se han incluido en la discusión sobre los efectos de la tecnología algunos trabajos que no están directamente relacionados con el mundo de la educación y que la autora había manejado con anterioridad, los criterios de inclusión que mayoritariamente hemos tenido en cuenta para escoger las fuentes de la actual revisión son:

- Cronología: artículos, libros y estudios publicados entre 2010 y 2021

- Idioma: español e inglés

- Campo de trabajo: educación

- Palabras clave: trabajos que contienen combinadas las palabras clave "Competencia digital", "Alfabetización digital", "desarrollo profesional docente", "Brecha ética digital”, "Tecnología educativa”, "Identidad digital” y sus equivalentes en inglés.

- Entorno geográfico: España y Latinoamérica

\section{OBJETIVOS}

Los objetivos de investigación que guían el estudio son los siguientes:

1. Problematizar el impacto y las consecuencias que tiene el uso y abuso de las tecnologías, internet y las redes sociales en las personas, y más en concreto en los niños y jóvenes que están en las aulas.

2. Abordar críticamente si la formación docente está desarrollando una visión adecuada de la identidad digital docente y de la competencia digital del profesorado, que pueda atender los desafíos descritos en el punto anterior.

3. Proponer, a modo de síntesis de lo debatido, algunas claves para mejorar la formación al profesorado en esta materia, asociadas a las áreas 5 y 6 del DigComEdu o Marco Europeo para la Competencia Digital de los Educadores (Redecker, 2020).

\section{FACTORES DE RIESGO Y CONSECUENCIAS EN EL USO Y ABUSO DE INTERNET, DE LA TECNOLOGÍA Y DE LAS REDES SOCIALES}

Cristóbal Cobo (2019) nos pide tomar una sana distancia de lo que él llama "tecnoentusiasmos imperantes" para poder practicar la desobediencia tecnológica y la reflexión crítica, en un momento en el que considera que hemos llegado al fin de la luna de miel digital. Internet ya no es una herramienta para el acceso universal y la inclusión, sino un instrumento de vigilancia, influencia, manipulación, "extractivismo" de datos y privacidad, pérdida de autocontrol y sobrecarga cognitiva. Varias décadas atrás en el tiempo, otras voces tecnocríticas como la de Michael Apple (1991) alegaban que el uso excesivo de tecnología educativa planteaba riesgos para la educación, entre los que destaca una distorsión de la enseñanza para favorecer a los docentes más expertos técnicamente en detrimento de profesores con otras habilidades pedagógicas, o planes de estudios que fomenten una mentalidad técnica, centrada en el "cómo", a expensas de una orientación humanista que se hace preguntas sobre los porqués. Nissenbaum y Walker (1998) también exploraban la preocupación general de muchos expertos en educación sobre la deshumanización de la escuela y las posibles 
Educando al homo digitalis: el papel de la educación y del digcomedu para paliar los efectos de los algoritmos, las fake news, la polarización y falta de pensamiento crítico

amenazas a los valores sociales y éticos resultantes de la informatización escolar. Se concretaba la preocupación en cuatro aspectos: que los estudiantes se apartaran de las personas y la sociedad; que la relación profesor-alumno pudiera romperse; que la enseñanza de valores importantes se viese comprometida; y que la educación se estandarizase en exceso. El trabajo concluía que había riesgos genuinos lo suficientemente graves para justificar la precaución, y que se debían tomar medidas para minimizarlos.

Exploraremos a continuación en qué medida estas preocupaciones se concretan en desafíos actuales en nuestras instituciones educativas, y cómo se relacionan con la identidad digital y la competencia digital docente.

\subsection{Sobreexposición: la construcción de la identidad a través de la tecnología}

Las instituciones educativas no están desconectadas de su contexto, sea ese ecosistema concebido a nivel local o global. La pandemia del COVID ha diluido más aún los límites entre la educación formal, no formal e informal, algo que ya se presentaba como deseable en el concepto del Long Life Learning planteado en el marco competencial educativo europeo que rige nuestro marco legal nacional y autonómico. La pandemia ha dejado claro que la escuela estaba presente allí donde un grupo de estudiantes tenía un dispositivo para conectarse con su profesorado y aprender, cada uno desde un lugar físico diferente. De hecho, este desdibujarse las fronteras es, según Eurídice Cabañes (2013) un rasgo de la posmodernidad, una hibridación de lo humano y lo tecnológico que se introduce en lo cotidiano, en nuestros modos de relacionarnos, que nos obliga a plantearnos constantemente hacia dónde nos dirigimos y cómo se construye la identidad individual y la colectiva. Cabañes huye de una visión tecnofóbica y nos invita a elegir si queremos ser "masa consumidora o colectivo creador". En la misma línea se expresa Dolors Reig (2016), con su ya clásico concepto de las tecnologías como TEP: tecnologías del empoderamiento y la participación.

Pero la omnipresencia de la tecnología también nos sobreexpone. El Papa Francisco ha hablado del "espectáculo constante de nuestras vidas hipervigiladas" en su última encíclica del 3 de octubre, Fratelli Tutti:

Todo se convierte en una especie de espectáculo que puede ser espiado, vigilado, y la vida se expone a un control constante. En la comunicación digital (...) el respeto al otro se hace pedazos y, de esa manera, al mismo tiempo que lo desplazo, lo ignoro y lo mantengo lejos, sin pudor alguno puedo invadir su vida hasta el extremo.

De forma similar se expresa José Carlos Ruiz, en su libro de 2021 Filosofía ante el desánimo:

No basta con conocerse a uno mismo, también hay que mostrarlo, exhibir nuestro mundo, narrar nuestros logros, contar nuestros anhelos, publicar nuestros sentimientos, hacer gala de nuestras opiniones, exponer nuestras creaciones, subir nuestras fotografías, «compartir» nuestras reflexiones... Todo

Vivat Academia. Revista de Comunicación. 2021, 154, 71-92 
Educando al homo digitalis: el papel de la educación y del digcomedu para paliar los efectos de los algoritmos, las fake news, la polarización y falta de pensamiento crítico

está orientado a huir de lo impersonal, que se percibe como sustracción, como negatividad. El problema aparece cuando nuestra personalidad, esa suma de temperamento (genético) y carácter (educacional), siente la presión de asumir una identidad exitosa, que por lo general está muy alejada de la realidad (p. 16).

Revisiones bibliográficas y estudios como el de Alonso-Ferreiro, Regueira y ZapicoBarbeitio (2019) han mostrado, además, que esa sobreexposición tiene efectos más perjudiciales cuando se trata de chicas adolescentes, y que el uso intenso de redes sociales lleva asociado en numerosas ocasiones prácticas que vulneran pilares básicos de la seguridad digital, como el sexting o el ciberacoso, una de las consecuencias más graves de la sobreexposición que estamos discutiendo.

La conexión a través de la tecnología es una nueva forma de compromiso intermedio entre la soledad y el miedo al fracaso o la invasión de la intimidad, algo que la profesora de Tecnología y Sociedad del MIT, Sherry Turkle, tilda de"esquizoide". Es mera ilusión, y cuando la ilusión del Otro se desvanece, nos sentimos más alienados que nunca. La profunda soledad que emana de la paradoja del "juntos, pero solos,", se desarrolla en su libro de 2012 Alone Together-Why we Expect More from Technology and Less from Each Other y define la nueva dimensión relacional del homo digitalis en estos tiempos en los que las redes sociales y la presencia virtual han suplantado en gran medida los encuentros físicos y el contacto humano "real", porque las pantallas permiten una separación de seguridad con la que evitamos lo más complicado de las relaciones "verdaderas" y el mostrarnos tal cual somos. Es una cuestión sobre la que ha profundizado aún más en su libro En defensa de la conversación (2020).

Otro de los filósofos de la era de la post-verdad y de los tecnocríticos del siglo XXI que han alertado de que conectividad virtual no es conexión auténtica es el coreano Byung-Chul Han. En su ensayo La expulsión de lo distinto (2017) alerta de que la hiperconexión digital no facilita el encuentro con otros, sino que nos aboca a encontrar personas que piensan igual que yo y se encargan de que nuestro horizonte de experiencias se vuelva cada vez más estrecho, haciéndonos pasar de largo ante los desconocidos o los que consideramos distintos. La consecuencia final es que la hiperconexión nos acaba encerrando en un inacabable bucle del yo y, en último término, nos llevan a una autopropaganda que nos adoctrina con nuestras propias nociones, mientras se refuerza nuestro sesgo de confirmación y nos hacemos más intolerantes con las ideas que nos interpelan y nos remueven.

El alumnado que habita nuestras aulas ve bastante clara la artificialidad de esta conexión virtual, y usa abundantemente el término "postureo", que se define en la RAE como "actitud artificiosa e impostada que se adopta por conveniencia o presunción”. El neologismo entró hace apenas cinco años en el diccionario de la Academia, en su 23 edición y, al menos en España, todos asociamos este vocablo a un comportamiento vanidoso y falso en las redes sociales. 
Educando al homo digitalis: el papel de la educación y del digcomedu para paliar los efectos de los algoritmos, las fake news, la polarización y falta de pensamiento crítico

\subsection{Sobreidentificación y FoMO: El miedo a quedarse fuera}

Aun reconociendo nuestros y nuestras jóvenes la inautenticidad que discutíamos en el apartado anterior, el tener conciencia de que muchos usuarios de las redes sociales "posturean" abundantemente y solo buscan aumentar su cifra de seguidores o de me gusta, lo cierto es que les aterroriza el "quedarse fuera" de su tribu virtual, se comparan todo el tiempo y permiten que su intimidad sea constantemente atropellada. Esta ansiedad psicológica derivada de la hiperconexión en redes sociales fue acuñada en 2013 con el acrónimo FoMO: Fear of Missing Out (Przybylski et al, 2013). Está relacionada con la teoría de la autodeterminación, de Ryan y Deci (2000), que afirma que el sentimiento de parentesco o conexión con los demás es una necesidad psicológica legítima que influye en la salud psicológica de los seres humanos. En este sentido, FoMO se entiende como un estado autorregulador que surge de la percepción situacional de cada persona. El estudio comprobó que existe una correlación significativa con tener baja autoestima, y aquellos que obtenían una menor satisfacción de las necesidades básicas, bajos niveles de estado de ánimo y satisfacción con la vida presentaban mayores niveles de FoMO, y presentan actitudes más compulsivas a la hora de revisar constantemente las redes sociales. Los resultados de la investigación de 2013 se han visto corroborados por otros estudios como el de Torres-Serrano (2020), que comprueba en su muestra con jóvenes usuarios de Instagram españoles de 18 a 30 años, que una alta puntuación en FoMO tiene correlación con un mayor uso de la red social, así como una puntuación elevada en FoMO correlaciona con una puntuación más alta en narcisismo, y por otro lado con una baja autoestima. El estudio de Franchina, Vanden Abeele, Van Rooij, Lo Coco y De Marez (2018) también ha evidenciado, esta vez con una muestra adolescentes flamencos, que una alta puntuación en la escala de FoMO es un predictor para posteriores comportamientos problemáticos en redes sociales, o de conflictos relacionales, en lo que se ya acuñado como "ningufoneo" o "phubbing": menospreciar a alguien que está físicamente presente, prestando más atención al Smartphone que a la persona delante de nosotros.

Pero incluso antes de que se acuñara el término FoMO, Echeburúa y de Corral (2010) alertan en su trabajo sobre las consecuencias del uso y abuso de Internet y de las redes sociales, y como ese abuso está relacionados con variables psicosociales, tales como la vulnerabilidad psicológica y el estrés, aconsejando estrategias preventivas en el seno de la familia y en las instituciones educativas. Para que estas estrategias de prevención sean efectivas, es evidente que el profesorado debe estar previamente formado en los riesgos que entraña un mal uso, algo que todavía es escaso en los planes de formación para la Competencia Digital Docente, más centrada en lo instrumental y en el diseño instruccional que en la "salud digital"; si bien, como recoge el estudio de AlonsoFerreiro, Regueira y Zapico-Barbeito (2019) se está incrementando el número de iniciativas, programas y planes que ponen el foco en la educación de la ciudadanía en un uso seguro y saludable de las tecnologías digitales. Algunos ejemplos son Pantallas Amigas; IS4K-Internet Segura for Kids; El Plan Navega con Rumbo y las guías legales en torno a las Redes sociales, menores de edad y privacidad en la red del INTECO.

Vivat Academia. Revista de Comunicación. 2021, 154, 71-92 
Educando al homo digitalis: el papel de la educación y del digcomedu para paliar los efectos de los algoritmos, las fake news, la polarización y falta de pensamiento crítico

\subsection{Desinformación: algoritmos, sesgos de confirmación, polarización y falta de pensamiento crítico}

En 2018, el estudio de los profesores del MIT Vosoughi, Roy y Aral, publicado en la revista Science, hizo saltar muchas alarmas sobre cuán impulsivo es el mundo adulto a la hora de compartir bulos online, con los consiguientes peligros que esto puede tener para la estabilidad de las democracias. El trabajo estudiaba la procedencia y el impacto de los bulos extendidos por Twitter. Lo más impactante de sus conclusiones es que no son los bots los que extienden las fake news, sino los seres humanos. El 70\% de las noticias falsas, sobre todo si son políticas, se viralizan mucho más rápido por Twitter que las verdaderas, lo cual incrementa el peligro de una sociedad cada vez más polarizada y manipulable, muy necesitada de una alfabetización digital crítica. Detrás del comportamiento del adulto que retuitea sin pensar hay cuestiones muy humanas: hacemos cada vez más caso de los pequeños influencers, y nuestra visceralidad, nuestros prejuicios y emociones se ven constantemente manipulados. Así, nuestro sesgo de confirmación es manejado al antojo de los algoritmos, que deciden qué debemos leer y cómo nos (des) informamos para polarizarnos más y más, y a la vez que la infodemia nos bombardea, las redes nos generan una adicción que impide el control inhibitorio del impulso, reflexión y un tiempo de reacción entre el estímulo y la respuesta que no nos conceden. De hecho, el inventor del "scroll infinito", Aza Raskin, ha comparado la adicción a las redes sociales con la dependencia de la cocaína (González, 2018).

El documental de Netflix "El dilema de las redes" (Orlowski, 2020) protagonizado por el mismo Aza Raskin y otros tecnólogos que crearon los gadgets y la inteligencia artificial cuya tarea principal es captar nuestra atención, denuncia también los riesgos de la sobrexposición a las redes sociales, la infodemia, la desinformación y el abuso de internet, además de avisar que se está poniendo en riesgo muy real la democracia y que nos puede llevar a un peligroso enfrentamiento social y a la destrucción de nuestras sociedades, mientras dejamos que los dispositivos e Internet aparentemente nos solucionen la vida y "piensen por nosotros".

Marina Garcés (2017) alerta de los peligros de creer demasiado en el "solucionismo" tecnológico. Se nos hace creer en la utopía de que la tecnología nos llevará a un mundo sin problemas, pero podemos acabar convertidos en humanos estúpidos que han delegado y "externalizado" su inteligencia y se dejan manejar por las máquinas y los algoritmos. Garcés considera que esta delegación es un gesto de pesimismo antropológico sin precedentes, y que estamos cayendo en el error de pensar que los algoritmos tomarán decisiones más informadas, en teoría más neutrales y libres del error humano, hasta que nos damos cuenta de que el algoritmo no es neutral. Harari (2018) también plantea este mismo dilema sobre el poder de los algoritmos en su libro 21 lecciones para el siglo XXI: hay posthumanistas convencidos de que ni siquiera les hace falta ya pensar críticamente y comprender la realidad que les rodea, porque todo son datos y lo fían todo a un buen sistema de información (Harari pone el ejemplo de los relojes inteligentes que recogen biorritmos y estado de salud, por ejemplo) o

Vivat Academia. Revista de Comunicación. 2021, 154, 71-92 
Educando al homo digitalis: el papel de la educación y del digcomedu para paliar los efectos de los algoritmos, las fake news, la polarización y falta de pensamiento crítico

algoritmos que parecen conocer mejor desde fuera mis gustos y necesidades que yo mismo y que les dicen a los niños, niñas y adolescentes qué vídeo de Youtube consumir después... Desde este artículo defendemos que este "dataísmo" a ultranza, que convierte en superfluo el pensamiento crítico, es un aspecto sobre el que la educación actual debe reflexionar largamente, porque puede convertirse en una nueva brecha en las estructuras de manipulación social y de poder: una élite pensante vs. una gran mayoría de personas que permiten que otros programen los algoritmos y piensen por ellos, o lo que Cristóbal Cobo llama "los vasallos de los datos, que sufren de infovulnerabilidad (Cobo, 2019, p.36). El autor problematiza lo que él llama el "hiperpositivismo digital":

¿la era de los datos masivos nos acerca a la verdad o es solamente un espejismo tecnofílico? (...) Es fundamental desarrollar habilidades a prueba de futuro, no limitadas a ciertas herramientas, instrumentos o metodologías (...) En definitiva, favorecer un desarrollo de nuevas capacidades que no están limitadas al aspecto técnico de determinadas herramientas tecnológicas, sino que favorezcan aprender a pensar de manera diferente y permitan enfrentar nuevos problemas desde una perspectiva que va más allá de la instrumental. (p. 29-30)

\subsection{Ceguera moral y ética}

Carrie James, miembro del Proyecto Zero de Harvard, publicó en 2014 su libro Disconnected: Youth, new media, and the ethics gap. En sus páginas James advertía que, a pesar de vivir en la era más informada de la humanidad- algo que debería en teoría favorecer una mayor amplitud de miras, más competencia global y un espíritu mayor de tolerancia a otras formas de entender el mundo y la realidad- la inclinación de los jóvenes actuales es a pensar de manera muy estrecha, a centrarse en ellos mismos y en gente muy cercana, conocida en la vida real. El libro se apoya en extensas entrevistas con niños y jóvenes cuya edad oscila entre los 10 y 25 años, y a través de estas entrevistas, James describe sus pensamientos sobre aspectos como la privacidad, la propiedad intelectual y la participación virtual. Se describen tres formas en que los jóvenes abordan su actividad online: practicar el pensamiento centrado en sí mismo, en que principalmente les inquietan las consecuencias para sí; el pensamiento moral, cuando se preocupan por las consecuencias que su praxis virtual tendrá para las personas que conocen; o el pensamiento ético, si se preocupan por personas desconocidas y comunidades más amplias. La conclusión de James es que los y las adolescentes, de forma mayoritaria, tienen ceguera ante las preocupaciones morales o éticas. Por ejemplo, el discurso hostil o los insultos, si se dan en contenidos online, se suele justificar con el comentario "es solo una broma". En cuanto a la propiedad intelectual, parecen sentir que "si está en red, es de todos"; muestran una actitud relajada ante la piratería, la descarga de archivos ajenos o el uso del copiar/pegar de materiales sin preocuparse por referenciar las fuentes.

Vivat Academia. Revista de Comunicación. 2021, 154, 71-92 
Educando al homo digitalis: el papel de la educación y del digcomedu para paliar los efectos de los algoritmos, las fake news, la polarización y falta de pensamiento crítico

\section{MARCOS PARA LA FORMACIÓN EN COMPETENCIA DIGITAL DOCENTE: ¿SE ESTÁ RESPONDIENDO A LOS DESAFÍOS ACTUALES?}

Como recuerdan Castañeda, Esteve y Adell (2018), la formación en tecnologías educativas supone todavía un reto tanto en la formación inicial, como en el desarrollo profesional permanente del docente. El profesorado parece ser muy consciente tanto de la necesidad de mejorar dicha formación, como de la necesidad de minimizar algunos de los riesgos para el alumnado que hemos discutido en epígrafes anteriores. El estudio de Pérez Escoda, Iglesias Rodríguez y Sánchez Gómez (2016) es uno de los que muestra más claramente la importancia que los maestros y futuros maestros españoles otorgan a la adecuada adquisición de la competencia digital docente y la necesidad que sienten de dominarla para su desempeño, lo que implica la necesidad de plantearla no solo en la formación inicial del profesorado, sino en la formación permanente. De modo similar, el $V$ estudio sobre el uso de la tecnología en educación realizado por Blink Learning (2019), con una muestra amplia de profesorado (1851 docentes en España y 1417 en Latinoamérica, entre los que el profesorado de Secundaria, Bachillerato y Formación Profesional alcanzaba un $74 \%$ de los encuestados) afirma que uno de los mayores retos del uso de la tecnología en el aula es el tema de la seguridad y protección de datos (22\%). Cuando se les pide identificar algún déficit académico en su alumnado al usar la tecnología, un $48 \%$ dice que sus estudiantes no pueden seleccionar y contrastar fuentes de información fiables, y un $30 \%$ dice que le preocupa la cuestión de la privacidad y protección de datos, y la incapacidad para percibir los riesgos a los que se exponen en la red.

La crisis del COVID-19 ha puesto más de relieve retos y problemas que ya existían, pero que se vieron agravados al tener que virtualizar durante meses la educación: escasa integración de la tecnología y las metodologías didácticas activas; baja competencia digital en un porcentaje significativo de docentes; la constatación de que la etiqueta de "nativos digitales" popularizada hace una década no responde a la realidad, porque el alumnado tampoco tiene una competencia digital alta a nivel de producción de contenidos. A todas estas cuestiones se suman, además, las brechas educativas de los estudiantes que proceden de entornos vulnerables, y que se han agrandado con la reciente brecha de acceso y de uso digital que ha hecho peligrar la continuidad en el proceso de aprendizaje durante la enseñanza remota de urgencia.

\subsection{Formación inicial docente, salud digital y alfabetización mediática: el caso de los Másteres de Formación al Profesorado}

Con el fin de determinar si los marcos de formación actuales están atendiendo a los retos de educar al Homo Digitalis, hemos comprobado si existen asignaturas dedicadas a explorar y problematizar la integración de las TIC dentro de la formación inicial al profesorado de Secundaria, por ser la franja de edad de ese alumnado más proclive a presentar los problemas que hemos señalado en el trabajo. Teniendo en cuenta que el SUE (Sistema Universitario Español) cuenta actualmente con 83 universidades, 50 públicas y 33 privadas, hemos consultado en Internet los planes de estudios de 20

Vivat Academia. Revista de Comunicación. 2021, 154, 71-92 
Teba Fernández, E.

Educando al homo digitalis: el papel de la educación y del digcomedu para paliar los efectos de los algoritmos, las fake news, la polarización y falta de pensamiento crítico

másteres de formación al profesorado de varias de esas universidades (la mayoría públicas, aunque hemos incluido en la consulta universidades privadas como Mondragón o la Camilo José Cela) para tener una muestra significativa. Solo en dos de los másteres consultados hay una asignatura obligatoria relacionada con la integración de las TIC y en otros cinco másteres, es optativa. Los créditos de esas asignaturas oscilan entre 2 y 4, de un total de 60. Si bien es cierto que los planes de estudios de estas titulaciones parten de la orden ECI/3858/2007, de 27 de diciembre, diez años antes de que existiera el Marco Digital del INTEF y trece años antes de que apareciera el DigComEdu, nos parece que la atención dedicada a la alfabetización y la salud digital en la formación inicial de los docentes de Secundaria es claramente insuficiente.

\begin{tabular}{|c|c|c|c|}
\hline & $\begin{array}{l}\text { Universidad } \\
\text { en la que se } \\
\text { imparte el } \\
\text { Máster } \\
\text { Formación del } \\
\text { Profesorado } \\
\text { de Secundaria }\end{array}$ & sitio web & $\begin{array}{l}\text { Asignatura dedicada a } \\
\text { la integración de las TIC } \\
\text { en Educación }\end{array}$ \\
\hline 1. & $\begin{array}{l}\text { Universidad } \\
\text { de Alicante }\end{array}$ & $\begin{array}{l}\text { https://web.ua.es/es/masteres/profesorado-de- } \\
\text { educacion-secundaria-obligatoria-y-bachillerato- } \\
\text { formacion-profesional-y-ensenyanza-de-idiomas/ }\end{array}$ & $\mathrm{NO}$ \\
\hline 2. & $\begin{array}{l}\text { Universitat } \\
\text { Autonoma de } \\
\text { Barcelona }\end{array}$ & $\begin{array}{l}\text { https://www.uab.cat/web/estudiar/la-oferta-de- } \\
\text { masteres-oficiales/plan-de-estudios/plan-de- } \\
\text { estudios-y-horarios/formacion-del-profesorado-de- } \\
\text { educacion-secundaria-obligatoria-y-bachillerato- } \\
\text { formacion-profesional-y-ensenanzas-de-idiomas- } \\
\text { 1096480309783.html?param1=1236065658068 }\end{array}$ & $\mathrm{NO}$ \\
\hline 3. & $\begin{array}{l}\text { Universidad } \\
\text { Camilo José } \\
\text { Cela }\end{array}$ & $\begin{array}{l}\text { https://www.ucjc.edu/estudio/master- } \\
\text { universitario-en-educacion-secundaria/ }\end{array}$ & $\begin{array}{l}\text { SÍ. OBLIGATORIA. } \\
\text { Tecnologías aplicadas a } \\
\text { la didácticas específicas- } \\
2 \text { créditos }\end{array}$ \\
\hline 4. & $\begin{array}{l}\text { Universidad } \\
\text { de Cantabria }\end{array}$ & $\begin{array}{l}\text { https:// web.unican.es/centros/educacion/estudio } \\
\text { s/asignaturas? } p=75 \& c=2021\end{array}$ & $\begin{array}{l}\text { SÍ: un apartado (Educar } \\
\text { en la sociedad } \\
\text { contemporánea: un } \\
\text { nuevo } \\
\text { escenario social y } \\
\text { educativo. La } \\
\text { importancia de las } \\
\text { nuevas tecnologías en la } \\
\text { escuela) dentro de la } \\
\text { asignatura "Familia y } \\
\text { Escuela en la Sociedad } \\
\text { de la Información" (4 } \\
\text { créditos) }\end{array}$ \\
\hline 5. & $\begin{array}{l}\text { Universidad } \\
\text { de Castilla La } \\
\text { Mancha }\end{array}$ & $\begin{array}{l}\text { https://www.uclm.es/estudios/masteres/master- } \\
\text { secundaria-fp-idiomas }\end{array}$ & $\mathrm{NO}$ \\
\hline
\end{tabular}

Vivat Academia. Revista de Comunicación. 2021, 154, 71-92 
Teba Fernández, E.

Educando al homo digitalis: el papel de la educación y del digcomedu para paliar los efectos de los algoritmos, las fake news, la polarización y falta de pensamiento crítico

\begin{tabular}{|c|c|c|c|}
\hline 6. & $\begin{array}{l}\text { Universidad } \\
\text { Complutense } \\
\text { de Madrid }\end{array}$ & $\begin{array}{l}\text { https://www.ucm.es/masterformacionprofesorad } \\
\text { o/plan-de-estudios }\end{array}$ & $\begin{array}{l}\text { NO (Solo en una } \\
\text { didáctica específica): } \\
\text { Herramientas TIC para } \\
\text { enseñanza de la } \\
\text { Informática y la } \\
\text { Tecnología }\end{array}$ \\
\hline 7. & $\begin{array}{l}\text { Universidade } \\
\text { da Coruña }\end{array}$ & https://estudos.udc.es/es/study/detail/437v06 & NO \\
\hline 8. & $\begin{array}{l}\text { Universidad } \\
\text { de } \\
\text { Extremadura }\end{array}$ & $\begin{array}{l}\text { https://www.unex.es/organizacion/servicios- } \\
\text { universitarios/servicios/servicio_becas/archivos/f } \\
\text { icheros/MASTERES\%20OFICIALES/planes-de- } \\
\text { estudio-anexo- } \\
\text { v/PlanMUFPESaclaracionpracticum.pdf }\end{array}$ & $\mathrm{NO}$ \\
\hline 9. & $\begin{array}{l}\text { Universidat de } \\
\text { les Illes } \\
\text { Balears }\end{array}$ & $\begin{array}{l}\text { https://estudis.uib.es/es/estudis-de- } \\
\text { master/master/MFPR/assignatures.html }\end{array}$ & $\mathrm{NO}$ \\
\hline 10. & $\begin{array}{l}\text { Universidad } \\
\text { de León }\end{array}$ & $\begin{array}{l}\text { https://www.unileon.es/estudiantes/oferta- } \\
\text { academica/masteres/mu-formacion-profesorado- } \\
\text { eso-bachillerato-fp-idiomas/plan-estudios }\end{array}$ & $\mathrm{NO}$ \\
\hline 11. & $\begin{array}{l}\text { Universidad } \\
\text { de Mondragón }\end{array}$ & $\begin{array}{l}\text { https://www.mondragon.edu/es/master- } \\
\text { universitario-habilitacion-docente-ejercicio- } \\
\text { profesiones-profesor-educacion-secundaria- } \\
\text { obligatoria-bachillerato-formacion-profesional- } \\
\text { ensenanza-idiomas/plan-estudios }\end{array}$ & $\begin{array}{l}\text { Sí, ES OPTATIVA } \\
\text { Tecnologías de la } \\
\text { Información y } \\
\text { Comunicación en la } \\
\text { Educación ( } 4 \text { créditos) }\end{array}$ \\
\hline 12. & $\begin{array}{l}\text { Universidad } \\
\text { de Murcia }\end{array}$ & $\begin{array}{l}\text { https://www.um.es/web/estudios/masteres/prof } \\
\text { esorado/historico-guias/2021-22 }\end{array}$ & $\begin{array}{l}\text { Sí, OPTATIVA. una } \\
\text { parte de la asignatura } \\
\text { “Actualización } \\
\text { Pedagógica I. } \\
\text { Metodología, Escenarios } \\
\text { Tecnológicos y } \\
\text { Evaluación Didáctica”- } \\
4 \text { créditos }\end{array}$ \\
\hline 13. & $\begin{array}{l}\text { UNED- } \\
\text { Universidad } \\
\text { Nacional de } \\
\text { Educación a } \\
\text { Distancia }\end{array}$ & $\begin{array}{l}\text { http://portal.uned.es/portal/page?_pageid=93,221 } \\
\underline{\text { 64976\&_dad=portal\&_schema=PORTAL\&idConten }} \\
\underline{\mathrm{ido}=8}\end{array}$ & $\mathrm{NO}$ \\
\hline 14. & $\begin{array}{l}\text { Universidad } \\
\text { de Navarra }\end{array}$ & https://www.unirioja.es/estudios/master/M0nA/ & $\mathrm{NO}$ \\
\hline 15. & $\begin{array}{l}\text { Universidad } \\
\text { de Oviedo }\end{array}$ & $\begin{array}{l}\text { https://www.uniovi.es/-/master-universitario-en- } \\
\text { formacion-del-profesorado-de-educacion- } \\
\text { secundaria-obligatoria-bachiller }\end{array}$ & $\begin{array}{l}\text { SÍ, OBLIGATORIA. } \\
\text { Tecnologías de la } \\
\text { Información y la } \\
\text { Comunicación: } 1 \text { crédito }\end{array}$ \\
\hline 16. & $\begin{array}{l}\text { Universidad } \\
\text { de la Rioja }\end{array}$ & https://www.unirioja.es/estudios/master/M0nA/ & $\mathrm{NO}$ \\
\hline 17. & $\begin{array}{l}\text { Universidad } \\
\text { de Sevilla }\end{array}$ & $\begin{array}{l}\text { https://www.us.es/estudiar/que-estudiar/oferta- } \\
\underline{\text { de-masteres/master-universitario-en-profesorado- }} \\
\underline{\text { de-ensenanza }}\end{array}$ & $\begin{array}{l}\text { SÍ, OPTATIVA. } \\
\text { Enseñanzas y } \\
\text { Aprendizaje con TIC-4 } \\
\text { créditos }\end{array}$ \\
\hline
\end{tabular}

Vivat Academia. Revista de Comunicación. 2021, 154, 71-92 
Teba Fernández, E.

Educando al homo digitalis: el papel de la educación y del digcomedu para paliar los efectos de los algoritmos, las fake news, la polarización y falta de pensamiento crítico

\begin{tabular}{|c|c|c|c|}
\hline 18. & $\begin{array}{l}\text { Universidad } \\
\text { de Valencia }\end{array}$ & $\begin{array}{l}\text { https://www.uv.es/uvweb/master-profesorado- } \\
\text { secundaria/es/programa-del-master/plan- } \\
\text { estudios/plan-estudios-1285886102408.html }\end{array}$ & $\mathrm{NO}$ \\
\hline 19. & $\begin{array}{l}\text { Universidad } \\
\text { de Valladolid }\end{array}$ & $\begin{array}{l}\text { https://www.uva.es/export/sites/uva/2.docencia } \\
\text { 2.02.mastersoficiales/2.02.01.ofertaeducativa/deta } \\
\text { lle/Master-en-Profesor-de-Educacion-Secundaria- } \\
\text { Obligatoria-y-Bachillerato-Formacion-Profesional- } \\
\text { y-Ensenanzas-de-Idiomas/ }\end{array}$ & $\mathrm{NO}$ \\
\hline 20. & $\begin{array}{l}\text { Universidad } \\
\text { de Zaragoza }\end{array}$ & https://estudios.unizar.es/estudio/ver?id=659 & $\begin{array}{l}\text { SÍ, OPTATIVA } \\
\text { Tecnologías de la } \\
\text { información y la } \\
\text { comunicación para el } \\
\text { aprendizaje. 3,5 créditos }\end{array}$ \\
\hline
\end{tabular}

Figura 1: Relación de másteres de formación al profesorado consultados y asignaturas dedicadas a la integración TIC.

\subsection{Formación permanente: la oferta en seguridad y salud digital del INTEF}

Dado que la seguridad de los y las escolares en el uso tecnología es una de las cuestiones que más preocupan a educadores y familias, desde el Ministerio de Educación y Formación Profesional, a través del Instituto Nacional de Tecnologías Educativas y Formación del Profesorado (INTEF), se han lanzado iniciativas para la formación del profesorado en ejercicio que buscan responder a varios de los retos que hemos apuntado en el presente trabajo. En la fecha en que escribimos el presente artículo, el INTEF oferta la primera edición de un curso NOOC (Nano Open Online Course) sobre Tecnoética, que dará comienzo en noviembre del 2021, y durante el año 2019 ha ofertado NOOCs sobre Cuidado de la identidad digital (Sexting y otras prácticas de riesgo), Ciberacoso, Respeto digital y protección de datos personales, medidas básicas de protección digital o Privacidad en la red.

\subsection{Marco Europeo para la Competencia Digital de los Educadores: el DigComEdu}

Más allá de los problemas circunstanciales descritos en secciones anteriores al discutir lo sucedido durante la crisis del COVID 19 y la "enseñanza remota de emergencia" (algunos de ellos, de hecho, se pueden considerar ya problemas coyunturales en entornos vulnerables) la competencia digital docente trasciende de la capacitación individual e instrumental del profesorado en materia de tecnología educativa, como remarcan Colás, Conde y Reyes (2019). En su trabajo analizan el Marco Digital Docente del INTEF del 2017, que consideran reduccionista en su planteamiento de la competencia digital docente, pues se centra fundamentalmente en habilidades técnicas y formales del profesor, y lo comparan con el Marco europeo DigCompEdu (Redecker, 2020). Los autores se decantan por este último marco, puesto que avanza desde el empoderamiento individual del docente en un mundo tecnológico hacia propuestas de transferencia del desarrollo de la competencia a los

Vivat Academia. Revista de Comunicación. 2021, 154, 71-92 
Educando al homo digitalis: el papel de la educación y del digcomedu para paliar los efectos de los algoritmos, las fake news, la polarización y falta de pensamiento crítico

estudiantes. Los últimos NOOCs ofrecidos por el INTEF desde 2019, y que hemos mencionado anteriormente, parecen querer corregir estas carencias formativas puestas de relieve en el estudio de Colás, Conde y Reyes (2019). Sería necesario, sin embargo, cuantificar el alcance real en porcentaje de docentes en ejercicio que se han matriculado en estos cursos para entender si este tipo de iniciativas puede contribuir de forma efectiva a minimizar los peligros que hemos planteado en el artículo.

Coincidimos con Colás, Conde y Reyes en que el DigComEdu lleva implícita una concepción de las competencias digitales como habilidades transformadoras y potenciadoras, sobre todo en las áreas 5 (Empoderamiento de los estudiantes) y 6 (Desarrollo de la competencia digital en los estudiantes) y se alinea mucho mejor con los marcos mundiales para la educación que mencionábamos en la introducción. El DigComEdu incide en que el profesorado tiene que capacitar al alumnado en la aplicación de las tecnologías digitales de forma crítica y responsable en cuanto a información, comunicación, generación de contenido, bienestar y resolución de problemas, y en ese sentido invita a que se den herramientas a los estudiantes para luchar contra los problemas que hemos discutido a lo largo del epígrafe 3 del presente estudio.

\subsection{La identidad digital docente}

Sin embargo, antes de poder acompañar en el desarrollo competencial del alumnado, los profesores debemos reflexionar sobre nuestra propia identidad digital docente, puesto que somos modelos de comportamiento para niños y jóvenes. Es vital la toma de conciencia de nuestro nuevo papel en la sociedad digital postmoderna, caracterizada por la hibridación, omnipresencia y corporeización de la tecnología de la que hablaba Cabañes (2013) y en la que nos convertimos en agentes activos que posibilitan experiencias de aprendizaje significativas, conectando e involucrando a los estudiantes y mejorando su desarrollo como aprendices.

La identidad digital docente, (...) es un proceso dinámico y permanente que implica dotar de sentido y reinterpretar las propias creencias, valores y experiencias a la luz de los nuevos contextos y marcos de relaciones en la sociedad contemporánea; un proceso de negociación, representación y constitución de la experiencia vivida dentro y fuera de la escuela por los docentes y sus visiones, creencias y expectativas sobre lo que significa ser docente y el tipo de docente que se quiere llegar a ser en una sociedad caracterizada por la digitalización de la experiencia humana, que frente a otros momentos históricos anteriores, ofrece nuevas formas de acceder al conocimiento y nuevas formas de representación, colaboración, de comunicación y de aprendizaje. (Correa et al., 2015. p. 47)

El aprendizaje y la enseñanza en las aulas modernas exigen enfoques pedagógicos para informar el diseño de entornos digitales que faciliten el aprendizaje conceptual y el desarrollo de la comprensión de los estudiantes sobre cómo realizar el aprendizaje y así ayudarles a desarrollar la competencia de aprender a aprender y el resto de

Vivat Academia. Revista de Comunicación. 2021, 154, 71-92 
Educando al homo digitalis: el papel de la educación y del digcomedu para paliar los efectos de los algoritmos, las fake news, la polarización y falta de pensamiento crítico

"destrezas del siglo XXI" (Engeness, 2021). Como enfatizan Colás, Conde y Reyes (2019), el docente digitalmente competente ya no es aquel que mejor sabe usar las tecnologías o el que mayor conocimiento tiene de las mismas, sino el que es capaz de aprovechar ese bagaje para el desarrollo de la competencia digital de sus propios estudiantes.

Castañeda, Esteve y Adell (2018) hacen hincapié en que los modelos institucionales de competencia digital docente que se habían usado antes del tienen varias carencias importantes: consideran la tecnología como una herramienta neutra en valores (cuando, como hemos discutido, su uso difícilmente puede ser considerado así) y además reducen la acción docente al trabajo en el aula, evitando de este modo dimensiones como el compromiso social y político o el posible impacto de las instituciones educativas en sus comunidades. Estamos de acuerdo con Castañeda, Esteve y Adell en que es necesaria una reconceptualización de la identidad digital docente que dirija a ambos, educador y educando, a un empoderamiento crítico y a la posibilidad de desarrollar su agencia, entendida como capacidad técnica guiada por el buen juicio, en mundo crecientemente mediado por la tecnología. Creemos que nos podemos apoyar en el área 5, y sobre todo en la 6, del DigComEdu, para llevar a cabo dicho empoderamiento crítico, y que las últimas actuaciones del INTEF en cuanto a oferta formativa son una buena señal de que se está tomando el camino correcto, si bien queda aún mucho por caminar, como muestra la escasa oferta en la formación inicial docente que hemos señalado en el epígrafe 5.1.

\subsection{Autopercepción de los futuros docentes sobre su competencia digital y formación inicial}

En el presente trabajo no hemos podido llevar a cabo una revisión sobre la autopercepción que los docentes españoles en activo tienen de su propia competencia digital, pero sí hemos localizado tres estudios que nos parece significativo nombrar sobre estudiantes de educación que mañana serán maestros y profesores. El primero, de Esteve-Mon, Gisbert-Cervera y Lázaro- Cantabrana (2016), realiza un cuestionario de autopercepción en estudiantes de educación, que arroja resultados esperanzadores: la amplia mayoría de los participantes en el estudio se consideraba bastante o muy capaz para el desempeño de la competencia digital. El estudio demostró que los estudiantes más jóvenes (20-24) mostraban una autoeficacia percibida mayor que el grupo de más edad, y las diferencias más significativas fueron, precisamente, las relativas a la ciudadanía digital y responsabilidad, lo que parece indicar que las nuevas generaciones son cada vez más conscientes de los riesgos que entraña el uso y abuso de internet, que comentábamos al inicio de este trabajo. Sin embargo, el trabajo de Moreno- Guerrero et al (2020) realizado sobre el marco del INTEF 2107 con estudiantes del Máster de Profesorado de Educación Secundaria Obligatoria, Bachillerato, Formación Profesional y Enseñanza de Idiomas en el Campus de Ceuta de la Universidad de Granada, no arroja resultados tan optimistas. Los grupos de futuros docentes tienen un nivel medio en el área competencia de información y alfabetización informacional, presentan valores más altos en el almacenamiento y recuperación de

Vivat Academia. Revista de Comunicación. 2021, 154, 71-92 
Educando al homo digitalis: el papel de la educación y del digcomedu para paliar los efectos de los algoritmos, las fake news, la polarización y falta de pensamiento crítico

información, datos y contenidos digitales; niveles medios en el almacenamiento y recuperación de información, de datos y contenidos digitales; pero tienen resultados más bajos en la evaluación de información, datos y contenidos digitales, precisamente uno de los aspectos que nos preocupaba en el análisis de este artículo.

El tercer trabajo (Alonso Ferreiro, 2018) describe una experiencia de ABP para el desarrollo de la competencia digital docente en la formación inicial del profesorado del Grado de Educación Infantil de la Universidad de Santiago de Compostela. Durante el ABP propuesto, los estudiantes universitarios debían elaborar colaborativamente un proyecto didáctico que integrara tareas con tecnologías digitales. La alta satisfacción expresada por el alumnado con la propuesta, sobre todo en lo que respecta a la posibilidad de implementar los ABP en sus visitas a las escuelas de prácticas, pone de manifiesto la importancia de las metodologías activas en la formación inicial del profesorado con respecto al desarrollo de la competencia digital docente y la capacidad de transferibilidad.

\section{CONCLUSIÓN: UN NUEVO ENFOQUE PARA LA FORMACIÓN Y EL DESARROLLO DE LA COMPETENCIA DIGITAL DOCENTE}

En el presente trabajo hemos discutido la necesidad de no quedarnos en una visión meramente instrumentalista de la Competencia Digital Docente si queremos atender los enormes desafíos de educar en un mundo cada vez más mediado por la tecnología. Tenemos una nueva oportunidad de concebir esta visión más amplia en los desarrollos curriculares de la nueva ley educativa LOMLOE, que señala la educación digital como uno de sus ejes transversales, pero que, sin embargo, solo ofertará una asignatura optativa en los tres primeros cursos de la ESO para el desarrollo de la competencia digital, como explicita en su artículo 24.

Nos parece importantísimo dar un enfoque más holístico a la praxis digital del docente y ayudar a empoderar a alumnado con una alfabetización más crítica e informada, que impida nuevas formas de exclusión o problemas psicosociales, con lo que sugerimos que se expliciten contenidos y criterios de evaluación que atiendan a estos aspectos para la totalidad de los desarrollos curriculares de la nueva ley educativa, de manera que sea tangible lo que manifiesta en su Disposición Final Cuarta la LOMLOE: "Las Administraciones educativas deberán incluir en el desarrollo del currículo la competencia digital a la que se refiere el apartado anterior, así como los elementos relacionados con las situaciones de riesgo derivadas de la inadecuada utilización de las TIC, con especial atención a las situaciones de violencia en la red."

Es imprescindible instruir a los niños y jóvenes de los riesgos que puede haber en la red, y ayudarles a llevar a cabo un uso responsable de los dispositivos y de su presencia en Internet, de cara a evitar consecuencias físicas y/o psicológicas negativas para ellos. Para lograrlo, se debe mejorar la formación inicial y permanente de los docentes, pero también es necesaria una profunda reflexión sobre nuestra identidad 
Educando al homo digitalis: el papel de la educación y del digcomedu para paliar los efectos de los algoritmos, las fake news, la polarización y falta de pensamiento crítico

digital y cómo influye sobre nuestro alumnado, dado que somos modelo de comportamiento que los estudiantes aprehenden.

Dentro del área 6 del DigComEdu (desarrollo de la competencia digital de los estudiantes), nos parece prioritario formar a los docentes para que puedan desarrollar a fondo el subapartado de información y alfabetización mediática, con indicadores tan importantes como "Analizar, comparar y evaluar de forma crítica la credibilidad y fiabilidad de las fuentes de datos, de la información y de los contenidos digitales", o el subapartado de comunicación y comunicación digital, con indicadores como "Ser consciente de las normas de comportamiento y de las habilidades para desenvolverse con tacto al utilizar las tecnologías digitales e interactuar en entornos digitales", "Adaptar las estrategias de comunicación a los destinatarios específicos y tener en cuenta la diversidad cultural y generacional en los entornos digitales" o, finalmente, "proteger la propia reputación". Tenemos por delante muchos retos importantes, entre los que destacan fomentar el pensamiento crítico de nuestro alumnado en entornos virtuales, y afianzar su autoimagen y autoestima.

El informe de la FAD Las TIC y su influencia en la socialización de adolescentes (Ballesteros y Picazo, 2019) mostró que las habilidades relacionadas con el diseño y creación de contenidos, pero sobre todo con la seguridad en Internet y uso adecuado de los dispositivos, eran las de menor relevancia en la enseñanza de los centros. Según los adolescentes entrevistados, el conocimiento sobre la manera de proteger los datos personales en Internet solo se enseñaba en el 30,5\% de los centros escolares, mientras que el desarrollo del pensamiento crítico sobre la información que se encuentra en Internet descendía a un $22,5 \%$, y el uso de dispositivos como tablets y smartphones se limitaba a un 18,6\%. En los próximos años se nos antoja urgente elevar estos porcentajes, si no queremos enfrentarnos a un aumento de los problemas que hemos discutido en este artículo.

\section{REFERENCIAS}

Alonso Ferreiro, A. (2018). Aprendizaje basado en proyectos para el desarrollo de la competencia digital docente en la formación inicial del profesorado. Relatec: Revista Latinoamericana de Tecnología Educativa, 17(1), 9-24.

Alonso-Ferreiro, A., Regueira, U., \& Zapico-Barbeito, M. H. (2019). Actitudes de alumnado preadolescente ante la seguridad digital: un análisis desde la perspectiva de género. Revista de Educación a Distancia (RED), 19(61).

Apple, M. W. (1991). The New Technology: Is It Part of the Solution or Part of the Problem in Education? Computers in the Schools, 8(1), 59-81.

Ballesteros, J. C., Picazo, L. (2019). Las TIC y su influencia en la socialización de adolescentes. Centro Reina Sofía sobre Adolescencia y Juventud, FAD.

Vivat Academia. Revista de Comunicación. 2021, 154, 71-92 
Teba Fernández, E.

Educando al homo digitalis: el papel de la educación y del digcomedu para paliar los efectos de los algoritmos, las fake news, la polarización y falta de pensamiento crítico

Bauman, Z. (2007). Tiempos líquidos. Barcelona: Tusquets Editores.

Bennis, W., \& Nanus, B. (1985). Organizational learning: The management of the collective self. New Management, 3(1), 6-13.

Blink Learning. (2019). V Estudio sobre el uso de la tecnología en la educación. https://www.realinfluencers.es/wp-content/uploads/2020/05/BLINK-informeTIC-2019-ESPA \%C3\%91A.pdf

Cabañes Martínez, E. (2013). De la hibridación al procomún: construyendo la realidad a través de la tecnología. Revista de Estudios de Juventud, (102), 9-23.

Castañeda, L., Esteve, F. y Adell, J. (2018). ¿Por qué es necesario repensar la competencia docente para el mundo digital? Revista de Educación a Distancia, 56, 120. https://doi.org/10.6018/red/56/6

Cobo, C. (2019). Acepto las Condiciones: Usos y abusos de las tecnologías digitales. Fundación Santillana.

Colás, P., Conde, J. y Reyes, S. (2019). El desarrollo de la competencia digital docente desde un enfoque sociocultural. Revista Comunicar, 61(V. XXVII), 2132. https://doi.org/10.3916/C61-2019-02

Correa Gorospe, J. M., Fernández Olaskoaga, L., Gutiérrez-Cabello Barragán, A, Losada Iglesias, D. y Ochoa-Aizpurúa Aguirre, B. (2015). Formación del Profesorado, Tecnología Educativa e Identidad Docente Digital. RELATEC: Revista Latinoamericana de Tecnología Educativa, 14 (1), 45-56.

Echeburúa, E. y de Corral, P. (2010). Adicción a las nuevas tecnologías y a las redes sociales en jóvenes: un nuevo reto. Adicciones, 22(2), 91-96.

Engeness, I. (2021) Developing teachers' digital identity: towards the pedagogic design principles of digital environments to enhance students' learning in the 21st century, European Journal of Teacher Education, 44(1), 96-114, https://doi.org/10.1080/02619768.2020.1849129

Esteve-Mon, F. M., Gisbert-Cervera, M. y Lázaro-Cantabrana, J. L. (2016). La competencia digital de los futuros docentes: ¿cómo se ven los actuales estudiantes de educación?. Perspectiva Educacional, Formación de Profesores, 55 (2), 38- 54.

Franchina, V., Vanden Abeele, M., Van Rooij, A. J., Lo Coco, G., \& De Marez, L. (2018). Fear of missing out as a predictor of problematic social media use and phubbing behavior among Flemish adolescents. International journal of environmental research and public health, 15(10), 2319. 
Educando al homo digitalis: el papel de la educación y del digcomedu para paliar los efectos de los algoritmos, las fake news, la polarización y falta de pensamiento crítico

Iglesia Católica. Papa (2013 -: Francisco) \& Francisco, P. (2020). Carta encíclica "Fratelli Tutti" sobre la fraternidad y la amistad social. http://www.vatican.va/content/francesco/es/encyclicals/documents/papafrancesco_20201003_enciclica-fratelli-tutti.html

Iglesia Católica. Papa (2013 -: Francisco) \& Francisco, P. (2020). Pacto Educativo Global. Instrumentum Laboris.

https://www.educationglobalcompact.org/resources/Risorse/instrumentumlaboris-sp.pdf

Garcés, M. (2017). Nueva ilustración radical. Editorial Anagrama.

González, G. (2018, 6 de julio). El inventor del scroll infinito compara las redes sociales con la cocaína. Gen Beta. https://www.genbeta.com/redes-sociales-ycomunidades/inventor-scroll-infinito-compara-redes-sociales-cocaina-searrepiente-haber-ayudado-que-sean-adictivas

Gordo López, Á. J., Rivera, J. D., Díaz Catalán, C., \& García Arnau, A. (2019). Factores de socialización digital juvenil. Estudio Delphi. Centro Reina Sofía sobre Adolescencia y Juventud. Fundación de Ayuda contra la Drogadicción (FAD).

Han, B. C. (2017). La expulsión de lo distinto. Herder Editorial.

Harari, Y. N. (2014). Sapiens. De animales a dioses: Una breve historia de la humanidad. Editorial Debate.

Harari, Y. N. (2016). Homo Deus: breve historia del mañana. Editorial Debate.

Harari, Y. N. (2018). 21 lecciones para el siglo XXI. Editorial Debate.

James, C. (2014). Disconnected: Youth, new media, and the ethics gap. MIT Press.

Moreno-Guerrero, A. J., Miaja-Chippirraz, N., Bueno-Pedrero, A., \& Borrego-Otero, L. (2020). El área de información y alfabetización informacional de la competencia digital docente. Revista Electrónica Educare, 24 (3), 521-536.

Nissenbaum, H., \& Walker, D. (1998). Will computers dehumanize education? A grounded approach to values at risk. Technology in society, 20(3), 237-273.

OECDE. (2018). The futures of education and skills. Education 2030. https:// www.oecd.org/education/2030/E2030\%20Position\%20Paper \% 20(05.04.2 $\underline{018) \cdot p d f}$ 
Teba Fernández, E.

Educando al homo digitalis: el papel de la educación y del digcomedu para paliar los efectos de los algoritmos, las fake news, la polarización y falta de pensamiento crítico

OECDE. (2020). Futures of education and skills 2030. https://www.oecd.org/education/2030-project/teaching-andlearning/learning/learning-compass-2030/

Orlowski, J. (Director). (2020). The Social Dilemma [Documental]. Netflix.

Overton, S. (2019, 26 de junio). Addicted and Overwhelmed: Why 1 in 3 UK Adults Are Cutting Back on Social Media. Exposure Ninja. https://exposureninja.com/blog/cutting-back-social-media/

Pérez-Escoda, A., Iglesias-Rodríguez, A., \& Sánchez-Gómez, M. C. (2016). Competencia digital y TIC: claves de la ciudadanía digital y requisitos para el futuro profesorado. Tecnología, innovación e investigación en los procesos de enseñanzaaprendizaje, 1793-1802.

Przybylski, A. K., Murayama, K., DeHaan, C. R., \& Gladwell, V. (2013). Motivational, emotional, and behavioral correlates of fear of missing out. Computers in human behavior, 29(4), 1841-1848.

Redecker, C. (2020). Marco Europeo para la Competencia Digital de los Educadores: DigCompEdu. Traducción al español de Fundación Universia e Instituto Nacional de Tecnologías Educativas y de Formación del Profesorado (INTEF). Ministerio de Educación y Formación Profesional. Gobierno de España.

Reig, D. (2016). TIC, TAC, TEP: Internet como escuela de vida. Cuadernos de pedagogía, (473), 24-27.

Ryan, R. y Deci, E. L. (2000). La Teoría de la Autodeterminación y la Facilitación de la Motivación Intrínseca, el Desarrollo Social, y el Bienestar. American Psychologist, 55(1), 68-78.

Ruiz, J. C. (2021). Filosofía ante el desánimo: pensamiento crítico para construir una personalidad sólida. Ediciones Destino.

Sánchez- Perera, G. y Andrada de Gregorio, G. (2013). Dispositivos, prótesis y artefactos de la subjetividad cyborg". Jóvenes, Tecnofilosofía y Arte Digital. Revista de Estudios de la juventud, 102, 41-54.

Terán Prieto, A. (2019) Ciberadicciones. Adicción a las nuevas tecnologías (NTIC). En AEPap (ed.). Congreso de Actualización Pediatría 2019. Madrid: Lúa Ediciones 3.0, 131141.

Torres Serrano, M. (2020). Fear of Missing Out (FoMO) y el uso de Instagram: análisis de las relaciones entre Narcisismo y Autoestima. Aloma, 38 (1), 31-38. 
Educando al homo digitalis: el papel de la educación y del digcomedu para paliar los efectos de los algoritmos, las fake news, la polarización y falta de pensamiento crítico

Turkle, S. (2017). Alone together: Why we expect more from technology and less from each other. Hachette UK.

Turkle, S. (2020). En defensa de la conversación: el poder de la conversación en la era digital. Ático de los Libros.

UNESCO. (2019a). Futures of education. Learning to Become. https://en.unesco.org/futuresofeducation/

UNESCO. (2019b). The initiative. Futures of Education. https://en.unesco.org/futuresofeducation/initiative

Vosoughi, S., Roy, D., \& Aral, S. (2018). The spread of true and false news online. Science, 359, 1146-1151. https://doi.org/10.1126/science.aap9559

World Economic Forum. (2020). The Future of Jobs Report 2020. World Economic Forum, Ginebra, Suiza.

\section{AUTOR/ES:}

\section{Eva Teba Fernández}

Doctora en Literaturas Hispánicas por Yale University; Master of Arts \& Master of Philosophy por Johns Hopkins University (EEUU). Experta en Educación Internacional y IB Certificate in Teaching and Learning por la Universidad Camilo José Cela, de Madrid. Cuenta con 16 años de recorrido docente e investigador en metodologías activas, gestión de la Calidad y los Recursos Humanos en instituciones educativas, y en el impacto producido por la transformación de espacios y escenarios de aprendizaje. Actualmente, es directora del Especialista en aprendizaje cooperativo e inteligencias múltiples, profesora en la Universidad Camilo José Cela de Madrid e investigadora en el equipo MEDEA, en esa misma universidad; cofundadora, socia y directora pedagógica de Educando Consultoría y vocal de la Junta de Gobierno del Colegio de Doctores y Licenciados de la Comunidad de Madrid.

Orcid ID: https://orcid.org/0000-0002-5887-3934 